\title{
Polarizabilities of Impurity Doped Quantum Dots under Pulsed Field: Role of Additive White Noise
}

\author{
Surajit Saha1, Manas Ghosh² \\ ${ }^{1}$ Department of Chemistry, Bishnupur Ramananda College, Bishnupur, India \\ ${ }^{2}$ Department of Chemistry, Physical Chemistry Section, Visva Bharati University, Santiniketan, India \\ Email: surajitchem11@gmail.com,pcmg77@rediffmail.com
}

Received 11 February 2015; accepted 25 March 2015; published 26 March 2015

Copyright $@ 2015$ by authors and Scientific Research Publishing Inc.

This work is licensed under the Creative Commons Attribution International License (CC BY). http://creativecommons.org/licenses/by/4.0/

(c) (i) Open Access

\section{Abstract}

We make a rigorous exploration of the profiles of a few diagonal and off-diagonal components of linear $\left(\alpha_{x x}, \alpha_{y y}, \alpha_{x y}\right.$ and $\left.\alpha_{y x}\right)$, first nonlinear $\left(\beta_{x x x}, \beta_{y y y}, \beta_{x y y}\right.$ and $\left.\beta_{y x x}\right)$, and second nonlinear $\left(\gamma_{x x x x}, \gamma_{y y y y}, \gamma_{x x y y}\right.$ and $\left.\gamma_{y y x x}\right)$ polarizabilities of quantum dots under the influence of external pulsed field. Simultaneous presence of additive white noise has also been considered. The quantum dot contains dopant described by a Gaussian potential. The numbers of pulse and the dopant location have been found to fabricate the said profiles jointly. The $\beta$ components display greater complexity in their profiles in comparison with the $\alpha$ and $\gamma$ counterparts. The presence of noise prominently enhances the influence of dopant coordinate on the polarizability profiles, particularly for $\alpha$ and $\gamma$ components. However, for $\beta$ components, the said influence becomes quite evident both in the presence and absence of additive noise. The study reveals some means of achieving stable, enhanced, and often maximized output of noise-driven linear and nonlinear polarizabilities.

\section{Keywords}

Quantum Dot, Impurity, Polarizability, Pulsed Field, Dopant Location, Additive White Noise

\section{Introduction}

The nonlinear optical effects displayed by Quantum dots (QDs) are enriched with much more subtleties than the bulk materials. As a result QDs have found a broad range of application in a variety of optical devices. Incorpo- 
ration of dopants to QDs causes a drastic change in the properties of the latter. The change happens because of the interplay between the intrinsic dot confinement potential and the dopant potential. We thus find a rich variety of useful investigations on doped QD [1]-[9]. From the perspective of optoelectronic applications, impurity driven modulation of linear and nonlinear optical properties is highly important in photodetectors and in several high-speed electro-optical devices [10]. Naturally, researchers have carried out a lot of important works on both linear and nonlinear optical properties of these structures [10]-[29].

External electric field often highlights important features arising out of the confined impurities. The electric field changes the energy spectrum of the carrier and thus influences the performance of the optoelectronic devices. In addition, the electric field often lifts the symmetry of the system and promotes emergence of nonlinear optical properties. Thus, the applied electric field possesses special importance in the field of research on the optical properties of doped QDs [30]-[42].

Recently we have made extensive investigations of the role of noise on the linear and nonlinear polarizabilities of impurity doped QDs [43]-[45]. In the present work we have explored some of the diagonal and off-diagonal components of linear $\left(\alpha_{x x}, \alpha_{y y}, \alpha_{x y}\right.$ and $\left.\alpha_{y x}\right)$, second order $\left(\beta_{x x x}, \beta_{y y y}, \beta_{x y y}\right.$ and $\left.\beta_{y x x}\right)$, and third order $\left(\gamma_{x x x x}, \gamma_{y y y y}, \gamma_{x x y y}\right.$ and $\left.\gamma_{y y x x}\right)$ polarizabilities of quantum dots in presence of Gaussian white noise introduced additively to the system. The doped system is exposed to an external pulsed electric field. The diagonal and off-diagonal components are expected to behave diversely because of their varied interactions with the pulsed field and noise. We have found that the number of pulses delivered to the system from the external field $\left(n_{p}\right)$ and the dopant coordinate $\left(r_{0}\right)$ contribute significantly in designing the various polarizability components. A change in $n_{p}$ in effect changes the amount of energy delivered to the doped system. And the role of dopant site has been given special importance following the notable works of Karabulut and Baskoutas [24], Baskoutas et al. [30], and Khordad and Bahramiyan [28] in the context of optical properties of doped heterostructures. The present enquiry addresses the important roles played by $n_{p}$ and $r_{0}$ in fabricating the various polarizability components in presence of additive noise.

\section{Method}

The Hamiltonian corresponding to a 2-d quantum dot with single carrier electron laterally confined (parabolic) in the $x-y$ plane and doped with a Gaussian impurity is given by

$$
H_{0}=H_{0}^{\prime}+V_{\text {imp }}
$$

where $H_{0}^{\prime}$ is the Hamiltonian in absence of impurity. Under the effective mass approximation it reads

$$
H_{0}^{\prime}=\frac{1}{2 m^{*}}\left[-i \hbar \nabla+\frac{e}{c} A\right]^{2}+\frac{1}{2} m^{*} \omega_{0}^{2}\left(x^{2}+y^{2}\right) .
$$

The confinement potential reads $V(x, y)=\frac{1}{2} m^{*} \omega_{0}^{2}\left(x^{2}+y^{2}\right)$ with harmonic confinement frequency $\omega_{0}$ and the effective mass $m^{*}$. The value of $m^{*}$ has been chosen to be $0.067 m_{0}$ resembling GaAs quantum dots. We have set $\hbar=e=m_{0}=a_{0}=1$ and perform our calculations in atomic unit. The parabolic confinement potential has been utilized in the study of optical properties of doped QDs by Çakir et al. [17] [18]. Recently Khordad and his coworkers introduced a new type of confinement potential for spherical QD’s called Modified Gaussian Potential, MGP [46] [47]. A perpendicular magnetic field $(\mathrm{B} \sim \mathrm{mT})$ serves as an additional confinement. In Landau gauge $\left[A=\left(B_{y}, 0,0\right)\right]$

( $A$ being the vector potential), the Hamiltonian transforms to

$$
H_{0}=-\frac{\hbar^{2}}{2 m^{*}}\left(\frac{\delta^{2}}{\delta x^{2}}+\frac{\delta^{2}}{\delta y^{2}}\right)+\frac{1}{2} m^{*} \omega_{0}^{2} x^{2}+\frac{1}{2} m^{*}\left(\omega_{0}^{2}+\omega_{c}^{2}\right) y^{2}-i \hbar \omega_{c} y \frac{\delta}{\delta x}
$$

$\omega_{c}=\frac{e B}{m^{*} c}$ being the cyclotron frequency and $2 \Omega=\omega_{0}^{2}+\omega_{c}^{2}$ can be viewed as the effective frequency in the $y$-direction. $V_{i m p}$ being the impurity (dopant) potential (Gaussian) [48]-[50] and is given by 


$$
V_{\text {imp }}=V_{0} e^{-\xi\left[\left(x-x_{0}\right)^{2}+\left(y-y_{0}\right)^{2}\right]}
$$

Positive values for $\xi$ and $V_{0}$ indicate a repulsive impurity. $V_{0},\left(x_{0}, y_{0}\right)$ and $\xi^{-1}$ represent the impurity potential, the dopant coordinate, and the spatial stretch of impurity, respectively.

We have employed a variational recipe to solve the time-independent Schrodinger equation and the trial function $\psi(x, y)$ has been constructed as a superposition of the product of harmonic oscillator eigenfunctions $\phi_{n}(p x)$ and $\phi_{m}(q y)$ respectively, as

$$
(x, y)=\sum_{n, m} C_{n, m} \phi_{n}(p x) \phi_{m}(q y)
$$

where $C_{n, m}$ are the variational parameters and $p=\sqrt{\frac{m^{*} \omega_{0}}{\hbar}}$ and $q=\sqrt{\frac{m^{*} \Omega}{\hbar}}$. In the linear variational calculation, requisite number of basis functions have been exploited after performing the convergence test. And $H_{0}$ is diagonalized in the direct product basis of harmonic oscillator eigenfunctions.

The external pulsed field can be represented by

$$
\varepsilon(t)=\varepsilon(0) S(t) \sin (v t)
$$

$\varepsilon(t)$ is the time-dependent field intensity modulated by a pulse-shape function $S(t)$ where the pulse has a peak field strength $\varepsilon(0)$, and a fixed frequency $v$. The pulsed field is applied along both $x$ and $y$ directions. In the present work we have invoked a sinusoidal pulse give by

$$
S(t)=\sin ^{2}\left(\frac{\pi t}{T_{p}}\right)
$$

where $T_{p}$ stands for pulse duration time. Thus $T_{p}$, or equivalently $n_{p}$ (the number of pulses), appears to be a key control parameter. Figure 1 depicts the profiles of five consecutive sinusoidal pulses as a function of time. With the application of pulsed field the time dependent Hamiltonian becomes

$$
H(t)=H_{0}+V_{1}(t)
$$

where

$$
V_{1}(t)=-|e|\left[\varepsilon_{x}(t) \cdot x+\varepsilon_{y}(t) \cdot y\right]
$$

In presence of additive white noise the time-dependent Hamiltonian becomes

$$
H(t)=H_{0}+V_{1}(t)+V_{2}(t)
$$

where $V_{2}(t)$ is the noise term $\sigma(t)$ that follows a Gaussian distribution with characteristics [43]-[45]:

$$
\langle\sigma(t)\rangle=0,
$$

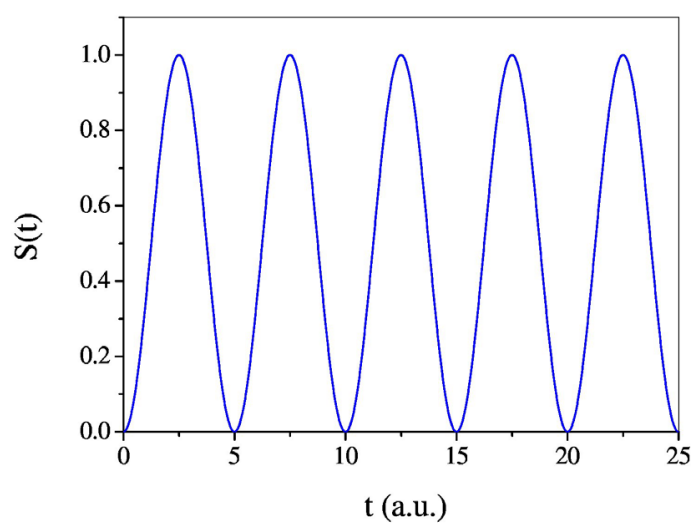

Figure 1. The sinusoidal pulse profile. 
and

$$
\left\langle\sigma(t) \sigma\left(t^{\prime}\right)\right\rangle=2 \mu \delta\left(t-t^{\prime}\right)
$$

where $\mu$ s tands for the noise strength.

The evolving wave function can now be described by a superposition of the eigenstates of $H_{0}$, i.e.

$$
\psi(x, y, t)=\sum_{q} a_{q}(t) \psi_{q}
$$

The time-dependent Schrödinger equation (TDSE) carrying the evolving wave function has now been solved numerically by 6-th order Runge-Kutta-Fehlberg method with a time step size $\Delta t=0.01$ a.u. after verifying the numerical stability of the integrator. The time dependent superposition coefficients $\left[a_{q}(t)\right]$ has been used to calculate the time-average energy of the dot $\langle E\rangle$. We have determined the energy eigenvalues for various combinations of field intensities and used them to compute some of the diagonal and off-diagonal components of linear and nonlinear polarizabilities by the following relations obtained by numerical differentiation. For linear polarizability.

$$
\alpha_{x x} \varepsilon_{x}^{2}=\frac{5}{2}\langle E(0)\rangle-\frac{4}{3}\left[\left\langle E\left(\varepsilon_{x}\right)\right\rangle+\left\langle E\left(-\varepsilon_{x}\right)\right\rangle\right]+\frac{1}{12}\left[\left\langle E\left(2 \varepsilon_{x}\right)\right\rangle+\left\langle E\left(-2 \varepsilon_{x}\right)\right\rangle\right]
$$

And a similar expression for $\alpha_{y y} \varepsilon_{y}^{2}$.

$$
\begin{aligned}
\alpha_{x y} \varepsilon_{x} \varepsilon_{y}= & \frac{1}{48}\left[E\left(2 \varepsilon_{x}, 2 \varepsilon_{y}\right)-E\left(2 \varepsilon_{x},-2 \varepsilon_{y}\right)-E\left(-2 \varepsilon_{x}, 2 \varepsilon_{y}\right)+E\left(-2 \varepsilon_{x},-2 \varepsilon_{y}\right)\right] \\
& -\frac{1}{3}\left[E\left(\varepsilon_{x}, \varepsilon_{y}\right)-E\left(\varepsilon_{x},-\varepsilon_{y}\right)-E\left(-\varepsilon_{x}, \varepsilon_{y}\right)+E\left(-\varepsilon_{x},-\varepsilon_{y}\right)\right],
\end{aligned}
$$

and a similar expression for computing $\alpha_{y x}$ component.

The components of first nonlinear polarizability (second order/quadratic hyperpolarizability) are calculated from following expressions

$$
\beta_{x x x} \varepsilon_{x}^{3}=\left[E\left(\varepsilon_{x}, 0\right)-E\left(-\varepsilon_{x}, 0\right)\right]-\frac{1}{2}\left[E\left(2 \varepsilon_{x}, 0\right)-E\left(-2 \varepsilon_{x}, 0\right)\right]
$$

and a similar expression is used for computing $\beta_{y y y}$ component.

$$
\beta_{x y y} \varepsilon_{x} \varepsilon_{y}^{2}=\frac{1}{2}\left[E\left(-\varepsilon_{x},-\varepsilon_{y}\right)-E\left(\varepsilon_{x}, \varepsilon_{y}\right)+E\left(-\varepsilon_{x}, \varepsilon_{y}\right)-E\left(\varepsilon_{x},-\varepsilon_{y}\right)\right]+\left[E\left(\varepsilon_{x}, 0\right)-E\left(-\varepsilon_{x}, 0\right)\right]
$$

and a similar expression for computing $\beta_{y x x}$ component.

The components of second nonlinear polarizability (third order/cubic hyperpolarizability) are given by

$$
\gamma_{x x x x} \varepsilon_{x}^{4}=4\left[E\left(\varepsilon_{x}\right)+E\left(-\varepsilon_{x}\right)\right]-\left[E\left(2 \varepsilon_{x}\right)+E\left(-2 \varepsilon_{x}\right)\right]-6 E(0)
$$

and a similar expression is used for computing $\gamma_{y y y y}$ component.

$$
\begin{aligned}
\gamma_{x x y y} \varepsilon_{x}^{2} \varepsilon_{y}^{2}= & 2\left[E\left(\varepsilon_{x}\right)+E\left(-\varepsilon_{x}\right)\right]+2\left[E\left(\varepsilon_{y}\right)+E\left(-\varepsilon_{y}\right)\right]-\left[E\left(\varepsilon_{x}, \varepsilon_{y}\right)+E\left(-\varepsilon_{x},-\varepsilon_{y}\right)+E\left(\varepsilon_{x},-\varepsilon_{y}\right)+E\left(-\varepsilon_{x}, \varepsilon_{y}\right)\right] \\
& -4 E(0),
\end{aligned}
$$

and a similar expression is used for computing $\gamma_{y y x x}$ component.

\section{Results and Discussion}

At the very onset of discussion it needs to be mentioned that the presence of additive noise changes the profiles of various polarizability components from that of noise-free condition. The magnitude of the components also increases invariably because of enhanced dispersive character of the system. However, in keeping with our previous findings a change in noise strength $(\mu)$ does not that much affect the outcomes [43]-[45]. 


\subsection{Linear $(\alpha)$ and Second Nonlinear $(\gamma)$ Polarizability Components}

Figure 2(a) depicts the profiles of $\alpha_{x x}$ component with variation of $n_{p}$ for on-center $\left(r_{0}=0.0\right.$ a.u.), near off-center $\left(r_{0}=28.28\right.$ a.u. $)$, and far off-center $\left(r_{0}=70.71\right.$ a.u. $)$ dopant locations, respectively. The plots exhibit different behaviors as $n_{p}$ is varied depending on the dopant location. For an on-center dopant $\alpha_{x x}$ minimizes at $n_{p} \sim 10$ [Figure 2(a) (i)] whereas for a near off-center dopant we observe maximization of the said component nearly at the same $n_{p}$ value [Figure 2(a) (ii)]. The profile takes a new pattern for a far off-center dopant when $\alpha_{x x}$ increases monotonically with $n_{p}$ up to $n_{p} \sim 10$ after which it saturates with further increase in $n_{p}$ [Figure 2(a) (iii)]. It therefore comes out that the interplay between $r_{0}$ and $n_{p}$ noticeably affects the profile of $\alpha_{x x}$ component and the interplay becomes most prominent at a typical pulse number of $n_{p} \sim 10$. The role of additive noise will be clear if we make a look at the said profile under noise-free condition. We have found that at that condition $\alpha_{x x}$ exhibits a profile similar to that of Figure 2(a) (iii) at all dopant locations. Thus, it can be inferred that the introduction of additive noise makes the role of $r_{0}$ more conspicuous. The other diagonal component $\alpha_{y y}$ evinces almost similar profile. Figure 2(b) displays the similar plot for the off-diagonal $\alpha_{x y}$ component. Firstly, we find a reduction (by a factor of $\sim 10^{2}$ ) in the value of $\alpha_{x y}$ in comparison with its diagonal counterpart. Moreover, the pattern of variation of the polarizability component shows considerable deviation from that of the diagonal one. For an on-center dopant $\alpha_{x y}$ falls steadily with increase in $n_{p}$ up to $n_{p} \sim 12$ beyond which it saturates [Figure 2(b) (i)]. The pattern gets changed with near and far off-center dopants while $\alpha_{x y}$ exhibits some initial steady behavior till $n_{p} \sim 5$ after which it rises considerably up to $n_{p} \sim 15$ followed by saturation thereafter [Figure 2(b) (ii) and Figure 2(b) (iii)]. As before, absence of additive noise downplays the role of dopant site. The absence makes $\alpha_{x y}$ profile look like that of Figure 2(b) (i) at all dopant locations. The off-diagonal $\alpha_{y x}$ component displays quite similar behavior.

Figure 3 depicts the similar profile for diagonal $\gamma_{x x x x}$ [(i) to (iii)] and off-diagonal $\gamma_{x x y y}$ [(iv) to (vi)] compnents. For on-center and near off-center dopants $\gamma_{x x x x}$ exhibits minima at $n_{p} \sim 10$ [Figure 3 (i) and Figure 3 (ii)].

However, for a far off-center dopant $\gamma_{x x x x}$ decreases smoothly up to $n_{p} \sim 15$ and settles thereafter [Figure 3 (iii)]. As we have observed for $\alpha_{x x}$, here also absence of additive noise scraps any influence of dopant site on the $\gamma_{x x x x}$ component. The other diagonal component $\gamma_{y y y y}$ behaves similarly. With an on-center dopant the off-diagonal $\gamma_{x x y y}$ component behaves quite similar to that of diagonal $\gamma_{x x x x}$ component with a far off-center dopant [Figure 3 (iv)]. With near and far off-center dopants $\gamma_{x x y y}$ exhibits a different behavior from that of oncenter one. In both these cases, the said component increases with $n_{p}$ steadily up to $n_{p} \sim 11$ beyond which they saturate [Figure 3 (v) and Figure 3 (vi)]. The other off-diagonal component $\gamma_{y y x x}$ does not show any appreciable alteration in its behavior. Interestingly, unlike the diagonal component, $\gamma_{x x y y}$ exhibits noticeable dependence on dopant site even in the absence of additive noise. However, the said dependence follows just the reverse pattern of what we have found here in the presence of noise [Figure 3 (iv)-(vi)].

\subsection{First Nonlinear $(\beta)$ Polarizability Components}

The inversion symmetry of the Hamiltonian [cf. Equation (3)] is preserved in the presence of an on-center dopant which annihilates the emergence of all $\beta$ components under noise-free condition. In absence of noise, the emergence of $\beta$ components has been observed only for off-center dopants. The additive noise changes the scenario and we find profiles of $\beta$ components at all dopant locations. However, the magnitude of the components enhances by a factor of $\sim 10^{5}$ for off-center dopants in comparison with the on-center analog. The additive noise, therefore, partially reduces the symmetry of the system.

Figure 4(a) represents the profiles of diagonal $\beta_{x x x}$ and $\beta_{y y y}$ components [(i) and (ii)] and off-diagonal $\beta_{x y y}$ and $\beta_{y x x}$ components [(iii) and (iv)] as a function of $n_{p}$ for an on-center dopant. $\beta_{x x x}$ and $\beta_{y y y}$ show minimization at $n_{p} \sim 10$ [Figure 4(a) (i)] and $n_{p} \sim 7$ [Figure 4(a) (ii)], respectively. $\beta_{x y y}$ exhibits steady behavior up to $n_{p} \sim 13$ [Figure 4(a) (iii)] and then rises prominently. $\beta_{y x x}$, on the other hand, rises smoothly till $n_{p} \sim 13$ and saturates henceforth [Figure 4(a) (iv)].

Figure 4(b) represents the similar plots for a near off-center dopant. $\beta_{x x x}$ has been found to decrease steadily up to $n_{p} \sim 10$ beyond which it saturates [Figure 4(a) (i)]. $\beta_{y y y}$ component exhibits maximization at $n_{p} \sim 6$ [Figure 4(a) (ii)]. $\beta_{x y y}$ displays a pattern resembling that of $\beta_{x x x}$ and saturates at $n_{p} \sim 18$ [Figure 4(a) (iii)]. $\beta_{y x x}$ component depicts a minimization at $n_{p} \sim 14$ [Figure 4(a) (iv)]. In absence of additive noise, we get somewhat different profiles for above $\beta$ components at the same dopant location. 


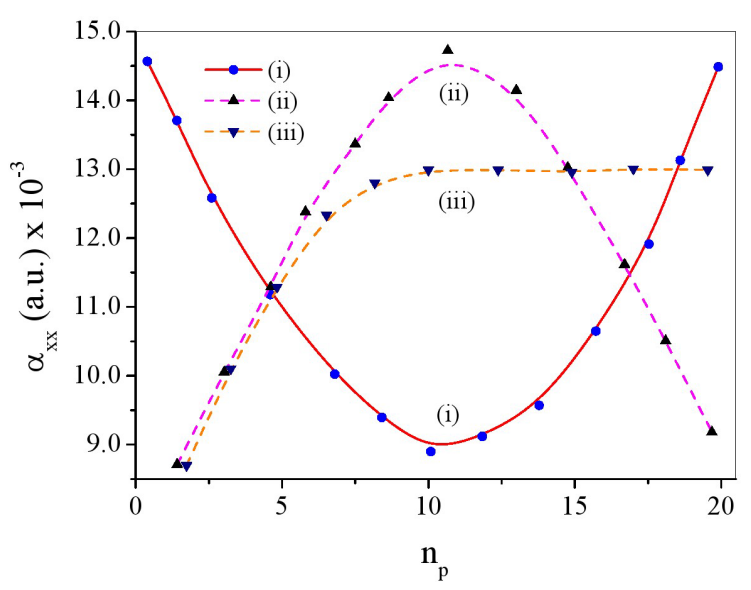

(a)

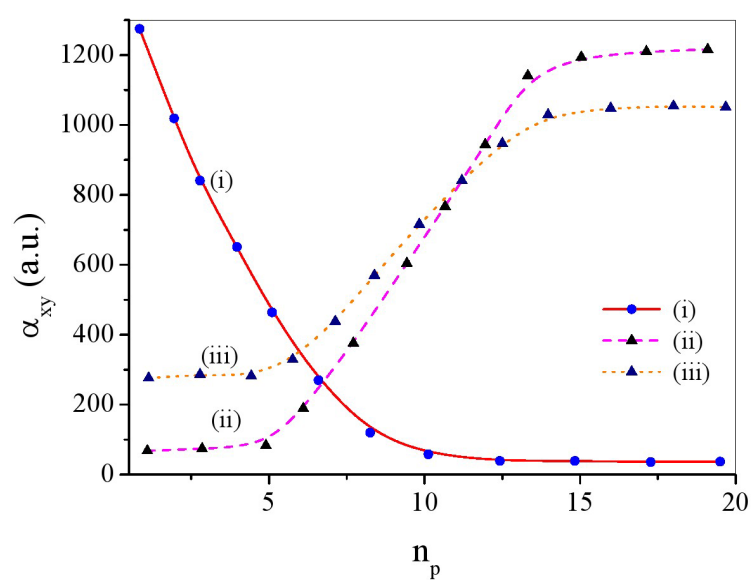

(b)

Figure 2. Plots of $\alpha$ components vs $n_{p}$ in presence of additive noise with (i) on-center, (ii) near off-center, and (iii) far off-center dopants: (a) for $\alpha_{x x}$ and (b) for $\alpha_{x y}$.

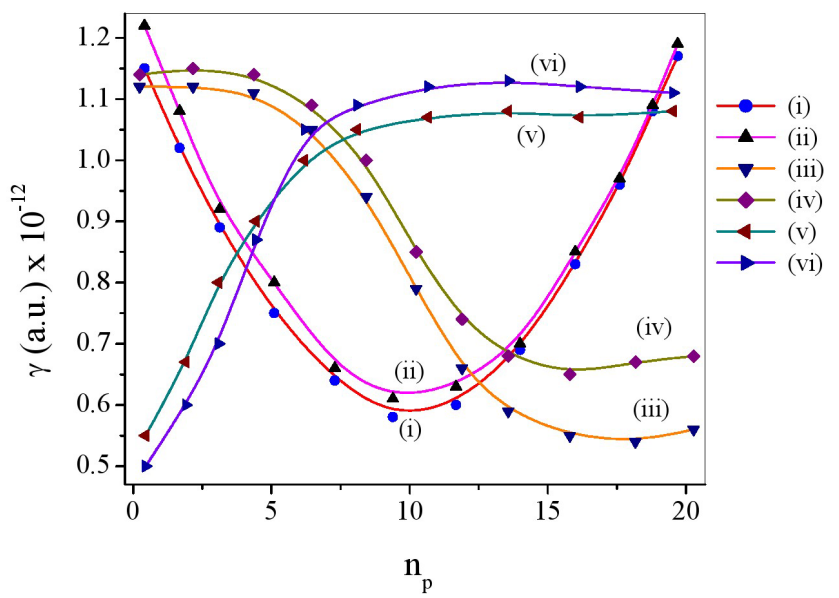

Figure 3. Plots of $\gamma$ components vs $n_{p}$ : (i) $\gamma_{x x x x}$ for on-center dopant; (ii) $\gamma_{x x x x}$ for near off-center dopant; (iii) $\gamma_{x x x x}$ for far off-center dopant; (iv) $\gamma_{x x y y}$ for on-center dopant; (v) $\gamma_{x x y y}$ for near off-center dopant; and (vi) $\gamma_{x x y y}$ for far offcenter dopant.

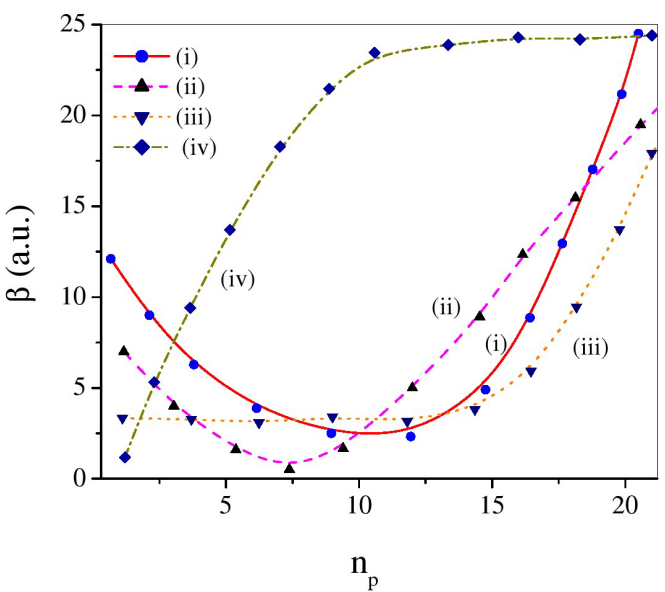

(a)

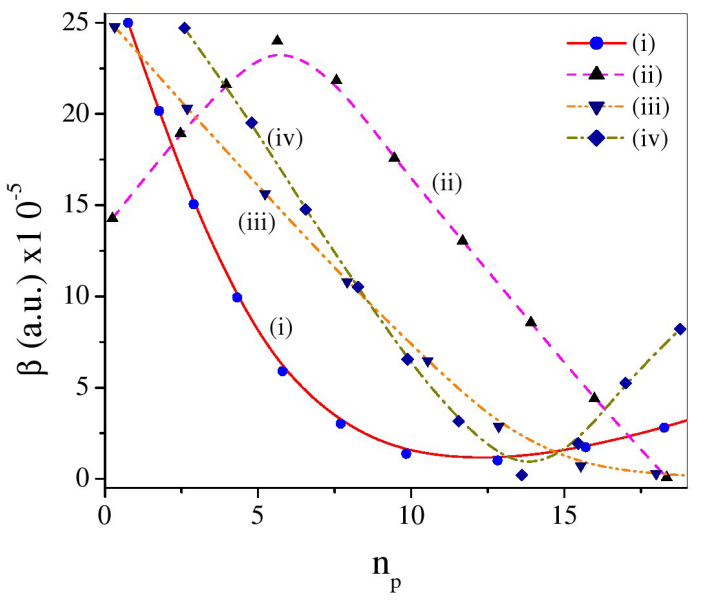

(b) 


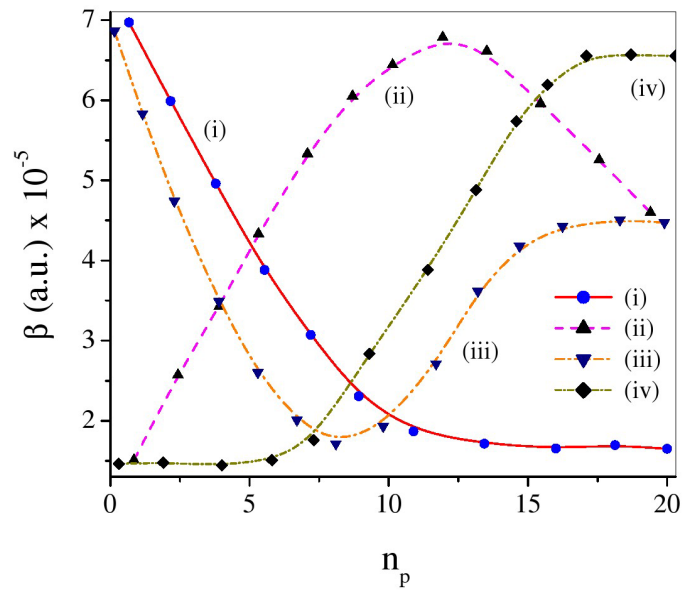

(c)

Figure 4. Plots of $\beta$ components vs $n_{p}$ : (a) on-center dopant, (b) near off-center dopant, and (c) far off-center dopant. (i) $\beta_{x x x}$, (ii) $\beta_{y y y}$, (iii) $\beta_{x y y}$, and (iv) $\beta_{y x x}$.

Figure 4(c) delineates the analogous plots for a far off-center dopant. $\beta_{x x x}$ depicts almost similar pattern [Figure 4(c) (i)] as in case of near off-center dopant; the only difference being that it now saturates at $n_{p} \sim 14$ (instead of $n_{p} \sim 10$ as in previous case). $\beta_{y y y}$ also, as before, exhibits maximization at $n_{p} \sim 12$ [Figure 4(c) (ii)] (instead of $n_{p} \sim 6$ as in previous case). $\beta_{x y y}$ component shows minimization at $n_{p} \sim 8$ [Figure 4(c) (iii)]. $\beta_{y x x}$ component initially exhibits a steady value up to $n_{p} \sim 6$, after which it rises sharply and culminates in saturation at $n_{p} \sim 18$ [Figure 4(c) (iv)].

Thus, it turns out that both dopant location and the number of pulses affect the polarizability profiles with sufficient delicacy. Particularly, the importance of dopant site in the present work complies with other notable works which manifest the contribution of dopant location in designing various properties of mesoscopic systems. In this context the works of Sadeghi and Avazpour [4] [5], Yakar et al. [7], Xie [9], Karabulut and Baskoutas [24], Khordad and Bahramiyan [28], and Baskoutas and his co-workers [30] deserve proper mention.

\section{Conclusion}

A few diagonal and off-diagonal components of linear, first nonlinear, and second nonlinear polarizabilities of impurity doped quantum dots have been explored under the influence of a pulsed field and in the presence of additive noise. The number of pulses fed into the system as well as the dopant location noticeably fabricates the polarizability profiles. It has been noticed that the $\beta$ components behave in a visibly different fashion from $\alpha$ and $\gamma$ components under pulsed field. Moreover, the $\beta$ components offer greater delicacy with variation of $n_{p}$ as well as dopant site $\left(r_{0}\right)$. The pulsed field thus modulates the second-order polarizability more sensitively than the linear and third-order polarizabilities. Whereas a variation in $n_{p}$ directly monitors the energy input from the external field, a varying $r_{0}$ modulates the spatial distribution of energy levels internally through different extents of dot-impurity interaction. Absence of additive noise diminishes the influence of dopant location on linear polarizability components; most prominently, on third-order polarizability components; somewhat less prominently. However, for second-order polarizability components, dopant location plays a significant role both in the presence and absence of additive noise, though in noticeably diverse manners. The study indicates some genuine pathways of achieving enhanced, maximized and often stable linear and nonlinear polarizabilities of doped QD in presence of additive noise which could be important in the field of noise-driven optical properties of these systems.

\section{References}

[1] Gülveren, B., Atav, Ü., Şahin, M. and Tomak, M. (2005) A Parabolic Quantum Dot with N Electrons and an Impurity. Physica E: Low-dimensional Systems and Nanostructures, 30, 143-149. http://dx.doi.org/10.1016/j.physe.2005.08.007

[2] Tąs, H. and Şahin, M. (2012) The Electronic Properties of Core/Shell/Well/Shell Spherical Quantum Dot with and 
without a Hydrogenic Impurity. Journal of Applied Physics, 111, Article ID: 083702.

[3] Baskoutas, S., Terzis, A.F. and Voutsinas E. (2004) Binding Energy of Donor States in a Quantum Dot with Parabolic Confinement. Journal of Computational and Theoretical Nanoscience, 1, 317-321. http://dx.doi.org/10.1166/jctn.2004.028

[4] Sadeghi, E. and Avazpour, A. (2011) Binding Energy of an Off-Center Donor Impurity in Ellipsoidal Quantum Dot with Parabolic Confinement Potential. Physica B: Condensed Matter, 406, 241-244. http://dx.doi.org/10.1016/j.physb.2010.10.051

[5] Sadeghi, E. (2009) Impurity Binding Energy of Excited States in Spherical Quantum Dot. Physica E: Low-Dimensional Systems and Nanostructures, 41, 1319-1322. http://dx.doi.org/10.1016/j.physe.2009.03.004

[6] Barati, M., Vahdani, M.R.K. and Rezaei, G. (2007) Lower-Lying States of Hydrogenic Impurity in Lens-Shaped and Semi-Lens-Shaped Quantum Dots. Journal of Physics: Condensed Matter, 19, Article ID: 136208.

[7] Yakar, Y., Çakir, B. and Özmen, A. (2013) Off-Center Hydrogenic Impurity in Spherical Quantum Dot with Parabolic Potential. Superlattices and Microstructures, 60, 389-397. http://dx.doi.org/10.1016/j.spmi.2013.05.015

[8] Akgül, S., Şahin, M. and KÖksal, K. (2012) A Detailed Investigation of the Electronic Properties of a Multilayer Spherical Quantum Dot with a Parabolic Confinement. Journal of Luminescence, 132, 1705-1713. http://dx.doi.org/10.1016/j.jlumin.2012.02.012

[9] Xie, W. (2008) Binding Energy of an Off-Center Hydrogenic Donor in a Spherical Gaussian Quantum Dot. Physica B: Condensed Matter, 403, 2828-2831. http://dx.doi.org/10.1016/j.physb.2008.02.017

[10] Vahdani, M.R.K. and Rezaei, G. (2009) Linear and Nonlinear Optical Properties of a Hydrogenic Donor in LensShaped Quantum Dots. Physics Letters A, 373, 3079-3084. http://dx.doi.org/10.1016/j.physleta.2009.06.042

[11] Rezaei, G., Vahdani, M.R.K. and Vaseghi, B. (2011) Nonlinear Optical Properties of a Hydrogenic Impurity in an Ellipsoidal Finite Potential Quantum Dot. Current Applied Physics, 11, 176-181. http://dx.doi.org/10.1016/j.cap.2010.07.002

[12] Xie, W. (2010) Impurity Effects on Optical Property of a Spherical Quantum Dot in the Presence of an Electric Field. Physica B: Condensed Matter, 405, 3436-3440. http://dx.doi.org/10.1016/j.physb.2010.05.019

[13] Karabulut, I. and Baskoutas, S. (2008) Linear and Nonlinear Optical Absorption Coefficients and Refractive Index Changes in Spherical Quantum Dots: Effects of Impurities, Electric Field, Size, and Optical Intensity. Journal of Applied Physics, 103, Article ID: 073512.

[14] Yakar. Y., Çakir. B., and Özmen A. (2010) Calculation of Linear and Nonlinear Optical Absorption Coefficients of a Spherical Quantum Dot with Parabolic Potential. Optics Communications, 283, 1795-1800. http://dx.doi.org/10.1016/j.optcom.2009.12.027

[15] Chen, T., Xie, W. and Liang, S. (2013) Optical and Electronic Properties of a Two-Dimensional Quantum Dot with an Impurity. Journal of Luminescence, 139, 64-68. http://dx.doi.org/10.1016/j.jlumin.2013.02.030

[16] Şahin, M. (2009) Third-Order Nonlinear Optical Properties of a One- and Two-Electron Spherical Quantum Dot with and without a Hydrogenic Impurity. Journal of Applied Physics, 106, Article ID: 063710.

[17] Çakir, B., Yakar, Y., Özmen, A., Özgür Sezer, M. and Şahin, M. (2010) Linear and Nonlinear Optical Absorption Coefficients and Binding Energy of a Spherical Quantum Dot. Superlattices and Microstructures, 47, 556-566. http://dx.doi.org/10.1016/j.spmi.2009.12.002

[18] Çakir, B., Yakar, Y. and Özmen, A. (2015) Linear and Nonlinear Optical Absorption Coefficient of Two-Electron Spherical Quantum Dot with Parabolic Potential. Physica B: Condensed Matter, 458, 138-143. http://dx.doi.org/10.1016/j.physb.2014.11.026

[19] Xie, W. (2008) Nonlinear Optical Properties of a Hydrogenic Donor Quantum Dot. Physics Letters A, 372, 5498-5500. http://dx.doi.org/10.1016/j.physleta.2008.06.059

[20] Baskoutas, S., Paspalakis, E. and Terzis, A.F. (2006) Effects of Excitons in Nonlinear Optical Rectification in Semiparabolic Quantum Dots. Physical Review B, 74, Article ID: 153306.

[21] Zeng, Z., Garoufalis, C.S., Terzis, A.F. and Baskoutas, S. (2013) Linear and Nonlinear Optical Properties of ZnS/ZnO Core Shell Quantum Dots: Effect of Shell Thickness, Impurity, and Dielectric Environment. Journal of Applied Physics, 114, Article ID: 023510. http://dx.doi.org/10.1063/1.4813094

[22] John Peter, A. (2006) Polarizabilities of Shallow Donors in Spherical Quantum Dots with Parabolic Confinement. Physics Letters A, 355, 59-62. http://dx.doi.org/10.1016/j.physleta.2006.01.107

[23] Xie, W. (2008) Linear and Nonlinear Optical Properties of a Hydrogenic Donor in Spherical Quantum Dots. Physica B: Condensed Matter, 403, 4319-4322. http://dx.doi.org/10.1016/j.physb.2008.09.021

[24] Karabulut, I. and Baskoutas, S. (2009) Second and Third Harmonic Generation Susceptibilities of Spherical Quantum Dots: Effects of Impurities, Electric Field and Size. Journal of Computational and Theoretical Nanoscience, 6, 153- 
156. http://dx.doi.org/10.1166/jctn.2009.1020

[25] Kumar, K.M., Peter, A.J. and Lee, C.W. (2012) Optical Properties of a Hydrogenic Impurity in a Confined $Z n_{1-x} C d_{x} S e / Z n S e$ Quantum Dot. Superlattices and Microstructures, 51, 184-193. http://dx.doi.org/10.1016/j.spmi.2011.11.012

[26] Tiutiunnyk, A., Tulupenko, V., Mora-Ramos, M.E., Kasapoglu, E., Ungan, F., Sari, H., SÖokmen, I. and Duque, C.A. (2014) Electron-Related Optical Responses in Triangular Quantum Dots. Physica E: Low-Dimensional Systems and Nanostructures, 60, 127-132. http://dx.doi.org/10.1016/j.physe.2014.02.017

[27] Yilmaz, S. and Şahin, M. (2010) Third-Order Nonlinear Absorption Spectra of an Impurity in a Spherical Quantum Dot with Different Confining Potential. Physica Status Solidi B, 247, 371-374. http://dx.doi.org/10.1002/pssb.200945491

[28] Khordad, R. and Bahramiyan, H. (2015) Impurity Position Effect on Optical Properties of Various Quantum Dots. Physica E: Low-Dimensional Systems and Nanostructures, 66, 107-115. http://dx.doi.org/10.1016/j.physe.2014.09.021

[29] Xie, W. and Liang, S. (2011) Optical Properties of a Donor Impurity in a Two-Dimensional Quantum Pseudodot. Physica B: Condensed Matter, 406, 4657-4660. http://dx.doi.org/10.1016/j.physb.2011.09.053

[30] Baskoutas, S., Paspalakis, E. and Terzis, A.F. (2007) Electronic Structure and Nonlinear Optical Rectification in a Quantum Dot: Effects of Impurities and External Electric Field. Journal of Physics: Condensed Matter, 19, Article ID: 395024.

[31] Lien, N.V. and Trinh, N.M. (2001) Electric Field Effects on the Binding Energy of Hydrogen Impurities in Quantum Dots with Parabolic Confinements. Journal of Physics: Condensed Matter, 13, 2563-2571. http://dx.doi.org/10.1088/0953-8984/13/11/312

[32] Murillo, G. and Porras-Montenegro, N. (2000) Effects of an Electric Field on the Binding Energy of a Donor Impurity in a Spherical GaAs(Ga,Al)As Quantum Dot with Parabolic Confinement. Physica Status Solidi B, 220, 187-190. http://dx.doi.org/10.1002/1521-3951(200007)220:1<187::AID-PSSB187>3.0.CO;2-D

[33] Kirak, M., Yilmaz, S., Şahin, M. and GenÇasian, M. (2011) The Electric Field Effects on the Binding Energies and the Nonlinear Optical Properties of a Donor Impurity in a Spherical Quantum Dot. Journal of Applied Physics, 109, Article ID: 094309. http://dx.doi.org/10.1063/1.3582137

[34] Duque, C.M., Mora-Ramos, M.E. and Duque, C.A. (2011) Hydrostatic Pressure and Electric Field Effects and Nonlinear Optical Rectification of Confined Excitons in Spherical Quantum Dots. Superlattices and Microstructures, 49, 264-268. http://dx.doi.org/10.1016/j.spmi.2010.06.008

[35] Duque, C.M., Barseghyan, M.G. and Duque, C.A. (2009) Hydrogenic Impurity Binding Energy in Vertically Coupled GaAsGa1-xAlxAs Quantum-Dots under Hydrostatic Pressure and Applied Electric Field. Physica B: Condensed Matter, 404, 5177-5180. http://dx.doi.org/10.1016/j.physb.2009.08.292

[36] Mora-Ramos, M.E., Duque, C.A., Kasapoglu, E., Sari, H. and Sökmen, I. (2012) Study of Direct and Indirect Exciton States in GaAsGa1-xAlxAs Quantum Dots under the Effects of Intense Laser Field and Applied Electric Field. The European Physical Journal B, 85, 312. http://dx.doi.org/10.1140/epjb/e2012-30148-5

[37] Narayanan, M. and John Peter, A. (2012) Electric Field Induced Exciton Binding Energy and Its Non-Linear Optical Properties in a Narrow InSb/InGaxSb1-x Quantum Dot. Superlattices and Microstructures, 51, 486-496. http://dx.doi.org/10.1016/j.spmi.2012.01.012

[38] López, S.Y., Porras-Montenegro, N. and Duque, C.A. (2009) Excitons in Coupled Quantum Dots: Hydrostatic Pressure and Electric Field Effects. Physica Status Solidi B, 246, 630-634. http://dx.doi.org/10.1002/pssb.200880536

[39] Kasapoglu, E., Ungan, F., Sari, H., Sökmen, I., Mora-Ramos, M.E. and Duque, C.A. (2014) Donor Impurity States and Related Optical Responses in Triangular Quantum Dots under Applied Electric Field. Superlattices and Microstructures, 73, 171-184. http://dx.doi.org/10.1016/j.spmi.2014.05.023

[40] Sadeghi, E. (2011) Electric Field and Impurity Effects on Optical Property of a Three-Dimensional Quantum Dot: A Combinational Potential Scheme. Superlattices and Microstructures, 50, 331-339. http://dx.doi.org/10.1016/j.spmi.2011.07.011

[41] Xie, W. and Xie, Q. (2009) Electric Field Effects of Hydrogenic Impurity States in a Disc-Like Quantum Dot. Physica B: Condensed Matter, 404, 1625-1628. http://dx.doi.org/10.1016/j.physb.2009.01.037

[42] Liang, S., Xie, W., Li, X. and Shen, H. (2011) Photoionization and Binding Energy of a Donor Impurity in a Quantum Dot under an Electric Field: Effects of the Hydrostatic Pressure and Temperature. Superlattices and Microstructures, 49, 623-631. http://dx.doi.org/10.1016/j.spmi.2011.03.013

[43] Ganguly, J. and Ghosh, M. (2014) Influence of Gaussian White Noise on the Frequency Dependent Linear Polarizability of Doped Quantum Dot. Chemical Physics, 438, 75-82. http://dx.doi.org/10.1016/j.chemphys.2014.04.014

[44] Ganguly, J. and Ghosh, M. (2014) Influence of Gaussian White Noise on the Frequency Dependent First Nonlinear 
Polarizability of Doped Quantum Dot. Journal of Applied Physics, 115, Article ID: 174313.

[45] Ganguly, J. and Ghosh, M. (2015) Exploring Static and Frequency-Dependent Third Nonlinear Polarizability of Doped Quantum Dots Driven by Gaussian White Noise. Physica Status Solidi B, 252, 289-297. http://dx.doi.org/10.1002/pssb.201451374

[46] Gharaati, A. and Khordad, R. (2010) A New Confinement Potential in Spherical Quantum Dots: Modified Gaussian Potential. Superlattices and Microstructures, 48, 276-287. http://dx.doi.org/10.1016/j.spmi.2010.06.014

[47] Khordad, R. (2013) Use of Modified Gaussian Potential to Study an Exciton in a Spherical Quantum Dot. Superlattices and Microstructures, 54, 7-15. http://dx.doi.org/10.1016/j.spmi.2012.10.014

[48] Adamowski, J., Kwaśniowski, A. and Szafran, B. (2005) LO-Phonon-Induced Screening of Electron-Electron Interaction in D- Centres and Quantum Dots. Journal of Physics: Condensed Matter, 17, 4489-4500. http://dx.doi.org/10.1088/0953-8984/17/28/008

[49] Bednarek, S., Szafran, B., Lis, K. and Adamowski, J. (2003) Modeling of Electronic Properties of Electrostatic Quantum Dots. Physical Review B, 68, Article ID: 155333.

[50] Szafran, B., Bednarek, S. and Adamowski, J. (2001) Parity Symmetry and Energy Spectrum of Excitons in Coupled Self-Assembled Quantum Dots. Physical Review B, 64, Article ID: 125301. 